\title{
Ring Expansion of Azetidinium Ylides: Rapid Access To the Pyrrolizidine Alkaloids Turneforcidine and Platynecine
}

\author{
John A. Vanecko and F. G. West* \\ Department of Chemistry, University of Utah, 315 S. 1400 East, Rm. 2020, Salt Lake \\ City, UT 84112-0850.
}

frederick.west@ualberta.ca

\section{Supporting Information}

\author{
Contents \\ Experimental Procedures........................ S-1-S \\ References............................................S-4 \\ NMR Spectra-....... S-5-S1
}

General information. Reactions were carried out in flame-dried glassware under a positive nitrogen atmosphere unless otherwise stated. Transfer of anhydrous solvents and reagents was accomplished with oven-dried syringes or cannulae. Solvents were distilled before use: methylene chloride from calcium hydride, tetrahydrofuran and diethylether from sodium/benzophenone ketyl, toluene from sodium metal. Ethereal diazomethane was prepared from Diazald according to literature procedures. ${ }^{1}$ Thin layer chromatography was performed on glass plates precoated with $0.25 \mathrm{~mm}$ Kieselgel $60 \mathrm{~F}_{254}$ (Merck). Flash chromatography columns were packed with 230-400 mesh silica gel (Merck). Radial chromatography was carried out on a Chromatotron 7924T (Harrison Research) with plates prepared using silica gel $60 \mathrm{~F}_{254}$ with gypsum binder (EM) on glass rotors. Products isolated by chromatographic purification are listed in order of increasing polarity. Gas chromatograms were obtained on a Hewlett-Packard 5890 series II capillary gas chromatograph with a 30 m HP-5 column and a flame ionization detector interfaced with a Hewlett-Packard 3396 series II integrator. The following parameters were used: injection port $220^{\circ} \mathrm{C}$, detector $250^{\circ} \mathrm{C}$. Two different programs were used: (1) initial temperature of $140{ }^{\circ} \mathrm{C}$, held for 2 min then a linear temperature ramp of $10^{\circ} \mathrm{C} / \mathrm{min}$ to $300{ }^{\circ} \mathrm{C}$, or (2) initial temperature of $120^{\circ} \mathrm{C}$, held for 2 min then a linear temperature ramp of $10{ }^{\circ} \mathrm{C} / \mathrm{min}$ to $300{ }^{\circ} \mathrm{C}$. Proton nuclear magnetic resonance spectra ( ${ }^{1} \mathrm{H}$ NMR) were recorded at $300 \mathrm{MHz}, 400 \mathrm{MHz}$ or $500 \mathrm{MHz}$. The chemical shifts are reported on the $\square$ scale $(\mathrm{ppm})$ and the spectra are referenced to tetramethylsilane $\left(\square=0 \mathrm{ppm},{ }^{1} \mathrm{H} ;{ }^{13} \mathrm{C}\right)$ or to deuteriochloroform $\left(\square=7.26 \mathrm{ppm},{ }^{1} \mathrm{H} ; \square=77.23 \mathrm{ppm}\right.$, ${ }^{13} \mathrm{C}$ ) as internal standard. Coupling constants $(J)$ are reported in Hertz $(\mathrm{Hz})$. Carbon nuclear magnetic resonance spectra $\left({ }^{13} \mathrm{C}\right.$ NMR) were recorded at $75 \mathrm{MHz}, 100 \mathrm{MHz}$ or $125 \mathrm{MHz}$ and are reported $(\mathrm{ppm})$ relative to the center line of the triplet from chloroform- $d$ (77.23 ppm). Infrared (IR) spectra were measured with a Mattson FTIR 3000 infrared spectrometer. Mass spectra were determined on a Finnigan Mat 95 high resolution gas chromatography/mass spectrometer with Finnigan Mat ICIS II operating system. 
Ethyl 3-Carboxyproline 3-Methyl Ester 8. To a solution of azetidine $5(0.099 \mathrm{~g}, 0.480 \mathrm{mmol})$, in $\mathrm{PhCH}_{3}(7.8 \mathrm{~mL})$ was added $\mathrm{Cu}(\mathrm{tfacac})_{2}(0.012 \mathrm{~g}, 0.032 \mathrm{mmol})$. The mixture was heated to reflux and a solution of ethyl diazoacetate $(0.030 \mathrm{~mL}, 0.32 \mathrm{mmol})$ in $\mathrm{PhCH}_{3}(1.3 \mathrm{~mL})$ was added via syringe pump over $12 \mathrm{~h}$. The resulting solution was cooled to r.t. and filtered through a plug of Celite using 30\% EtOAc/hexanes. Concentration of the filtrate provided a yellow oil which was purified by radial chromatography $(2 \mathrm{~mm}$ silica gel plate; 3:7 EtOAc/hexanes collecting $10 \mathrm{~mL}$ fractions). Collection and concentration of the product containing fractions provided $0.076 \mathrm{~g}$ $(82 \%)$ of 8 as a yellow oil (1:1 mixture of diastereomers as determined by integration of the OMe singlets in the ${ }^{1} \mathrm{H}$ NMR spectrum): $\mathrm{R}_{f}=0.17$ (2:3 EtOAc/hexanes); IR (thin film) 2979, 2954, 2839, 1740, 1603, 1495, $1453 \mathrm{~cm}^{-1}$; ${ }^{1} \mathrm{H}$ NMR (300 MHz, $\left.\mathrm{CDCl}_{3}\right) \delta$ 7.34-7.22 (m, 10H); 4.21-4.09 $(\mathrm{m}, 4 \mathrm{H}) ; 3.99(\mathrm{~d}, J=12.9 \mathrm{~Hz}, 1 \mathrm{H})$; 3.83-3.70 (m, 3H); $3.71(\mathrm{~s}, 3 \mathrm{H}), 3.65(\mathrm{~s}, 3 \mathrm{H}), 3.58(\mathrm{~d}, J=2.9$ $\mathrm{Hz}, 1 \mathrm{H}), 3.54(\mathrm{~d}, J=3.6 \mathrm{~Hz}, 1 \mathrm{H}), 3.33-3.21(\mathrm{~m}, 2 \mathrm{H}), 3.06-2.98(\mathrm{~m}, 2 \mathrm{H}), 2.71$ (ddd, $J=8.8,7.4$, $7.4 \mathrm{~Hz}, 1 \mathrm{H}$ ), 2.56-2.47 (m, 1H), 2.35 (dddd, $J=12.7,9.2,9.2,7.4 \mathrm{~Hz}, 1 \mathrm{H}), 2.22-2.02(\mathrm{~m}, 3 \mathrm{H})$, 1.26 (t, $J=7.2 \mathrm{~Hz}, 3 \mathrm{H}), 1.25$ (t, $J=7.2 \mathrm{~Hz}, 3 \mathrm{H}) ;{ }^{13} \mathrm{C}$ NMR $\left(75 \mathrm{MHz}, \mathrm{CDCl}_{3}\right) \delta 174.5,172,8$, $172.7,171.5,138.4,138.4,129.2,129.1,128.4,128.4,127.4,127.3,68.4,66.2,61.2,60.7,58.8$, $57.2,52.8,52.4,52.1,51.5,47.3,46.6,27.7,26.7,14.5,14.4$.

Methyl Azetidine-2-carboxylate. Palladium on carbon $(0.525 \mathrm{~g}, 10 \%$ active) was carefully added to a flask containing azetidine $5(0.525 \mathrm{~g}, 2.40 \mathrm{mmol})$ under argon. Ammonium formate $(0.453 \mathrm{~g}, 7.20 \mathrm{mmol})$ was then added followed by slow addition of $\mathrm{MeOH}(12 \mathrm{~mL})$ via syringe. The resulting mixture was then heated to reflux. Upon complete consumption of starting material as judged by TLC (ca. $45 \mathrm{~min}$ ) the mixture was cooled to r.t., filtered through filter paper (Whatman \#1), and washed with $\mathrm{CH}_{2} \mathrm{Cl}_{2}(50 \mathrm{~mL})$. Pentane $(50 \mathrm{~mL})$ was then added and the solvent was carefully removed under reduced pressure while maintaining a water bath temperature of $0^{\circ} \mathrm{C}$ until approximately $10 \mathrm{~mL}$ of solvent was left. Pentane $(50 \mathrm{~mL})$ was added and the solvent carefully removed once again until $10 \mathrm{~mL}$ of solvent was left. The resulting solvent was removed by blowing a steady stream of argon to provide the crude $\mathbf{3 7}$ as a colorless, volatile oil which was used immediately in the next reaction: $\mathrm{R}_{f}=0.21$ (1:4 $\mathrm{MeOH} / \mathrm{CH}_{2} \mathrm{Cl}_{2}$ ); IR (thin film) 3331, 3245, 3167, 2960, 2888, 2352, 2252, $1733 \mathrm{~cm}^{-1} ;{ }^{1} \mathrm{H}$ NMR (500 $\left.\mathrm{MHz}, \mathrm{CDCl}_{3}\right) \delta 4.31(\mathrm{dd}, J=9.2,6.0 \mathrm{~Hz}, 1 \mathrm{H}), 3.77(\mathrm{~s}, 3 \mathrm{H}), 3.66-3.61(\mathrm{~m}, 1 \mathrm{H}), 3.57-3.53(\mathrm{~m}, 1 \mathrm{H})$, 2.72 (dddd, $J=11.3,8.7,8.7,6.5 \mathrm{~Hz}, 1 \mathrm{H}$ ), 2.43 (br s, 1H), 2.38 (dddd, $J=12.0,8.9,6.2,6.2 \mathrm{~Hz}$, $1 \mathrm{H}) ;{ }^{13} \mathrm{C}$ NMR $\left(125 \mathrm{MHz}, \mathrm{CDCl}_{3}\right) \delta 175.5,58.7,52.3,45.2,25.7$. These data were consistent with the previously reported values for this compound. ${ }^{2}$

Methyl 1-(3'-Oxo-4'-diazo)butylaziridine-2-carboxylate 1a. To crude methyl azetidine-2carboxylate prepared via the preceding method $(0.275 \mathrm{~g}, 2.40 \mathrm{mmol})$ was added freshly prepared bromide $10(0.212 \mathrm{~g}, 1.20 \mathrm{mmol}){ }^{3}$ The flask was then flushed with argon and acetonitrile $(12 \mathrm{~mL})$ was added followed by $N, N$-diisopropylethyl amine $(0.23 \mathrm{~mL}, 1.3 \mathrm{mmol})$ via syringe and the resulting mixture was allowed to stir under argon at r.t. for $16 \mathrm{~h}$. The mixture was concentrated under reduced pressure and then purified by flash column chromatography (silica gel; $1.5 \mathrm{~cm} \times 8.0 \mathrm{~cm}$ column collecting $4 \mathrm{~mL}$ fractions; $1: 1 \mathrm{EtOAc} /$ hexanes $(40 \mathrm{~mL})$ followed by $1: 9$ $\mathrm{MeOH} / \mathrm{EtOAc}(100 \mathrm{~mL}))$. The product-containing fractions were collected and concentrated under reduced pressure to provide $0.264 \mathrm{~g}\left(96 \%\right.$, two steps) of diazoketone $1 \mathrm{a}$ as a yellow oil: $\mathrm{R}_{f}=$ $0.63\left(20 \% \mathrm{MeOH} / \mathrm{CH}_{2} \mathrm{Cl}_{2}\right)$; IR (thin film) 3116, 2954, 2845, 2108, 1737, $1637 \mathrm{~cm}^{-1} ;{ }^{1} \mathrm{H}$ NMR (500 $\left.\mathrm{MHz}, \mathrm{CDCl}_{3}\right) \delta 5.52(\mathrm{br} \mathrm{s}, 1 \mathrm{H}), 3.73(\mathrm{~s}, 3 \mathrm{H}), 3.68(\mathrm{dd}, J=8.7,8.7 \mathrm{~Hz}, 1 \mathrm{H}), 3.40$ (dddd, $J=8.7$, 6.2, 2.5, 0.8 Hz, 1H), $2.91(\mathrm{~m}, 2 \mathrm{H}), 2.76(\mathrm{~m}, 1 \mathrm{H}), 2.42(\mathrm{br} \mathrm{s}, 2 \mathrm{H}), 2.34(\mathrm{~m}, 1 \mathrm{H}), 2.25$ (dddd, $J=$

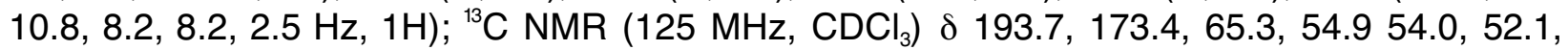
51.3, 39.2, 21.7; HRMS (Cl) calcd for $\mathrm{C}_{9} \mathrm{H}_{14} \mathrm{~N}_{3} \mathrm{O}_{3}\left(\mathrm{MH}^{+}\right) \mathrm{m} / \mathrm{z} 212.0957$, found $\mathrm{m} / \mathrm{z} 212.1024$.

Pyrrolizidine Esters $3 a$ and 4a. To a stirring solution of $\mathrm{Cu}(\mathrm{acac})_{2}(0.004 \mathrm{~g}, 0.02 \mathrm{mmol})$ in 
degassed toluene $(1.6 \mathrm{~mL})$ at $85{ }^{\circ} \mathrm{C}$ was added a solution of diazoketone $5(0.033 \mathrm{~g}, 0.16 \mathrm{mmol})$ in degassed toluene $(0.16 \mathrm{~mL})$ dropwise over $5 \mathrm{~min}$. Upon complete addition, the syringe was washed with toluene $(0.2 \mathrm{~mL})$ and the contents were added directly to the reaction. The resulting solution was allowed to cool to rt and concentrated under reduced pressure to provide a brown oil. The oil was immediately purified by flash column chromatography using a $1.5 \mathrm{~cm} \times 8.0 \mathrm{~cm}$ column eluting with a gradient of $35 \mathrm{~mL}$ each $2 \%, 6 \%$, and $12 \% \mathrm{MeOH} / \mathrm{CH}_{2} \mathrm{Cl}_{2}$ collecting $2 \mathrm{~mL}$ fractions. The product-containing fractions were collected and concentrated under reduced pressure to give $0.019 \mathrm{~g}(80 \%)$ of an inseparable $3.6: 1$ mixture (GC) of $3 \mathbf{a}$ and $4 \mathbf{a}$ as a brown oil whose physical data agreed with literature values. ${ }^{4}$

Hydroxyester 11 and Lactone 12. To a solution of pyrrolizidines 7 and 8 (0.062g, $0.34 \mathrm{mmol})$ in dry $\mathrm{MeOH}(4.5 \mathrm{~mL})$ was added $\mathrm{PtO}_{2}(0.031 \mathrm{~g})$. The resulting mixture was then hydrogenated ${ }^{4 b}$ at $\mathrm{rt}$ and atmospheric pressure for $4 \mathrm{~h}$, at which time it was filtered through a pipet containing glass wool. The pipet was washed with $10 \mathrm{~mL}$ of a $1: 1$ mixture of $\mathrm{MeOH} / \mathrm{CH}_{2} \mathrm{Cl}_{2}$ and the resulting filtrate concentrated under reduced pressure. The resulting yellow oil was then purified by flash column chromatography on neutral alumina using a $1.5 \mathrm{~cm} \times 8.0 \mathrm{~cm}$ column eluting with a gradient of $75 \mathrm{~mL}$ each of $70 \%$ and $100 \%$ EtOAc/hexanes followed by $75 \mathrm{~mL}$ of $3: 1$ EtOAc/MeOH collecting $2 \mathrm{~mL}$ fractions. The product-containing fractions were collected and concentrated under reduced pressure to provide $0.043 \mathrm{~g}$ of 11 and $0.008 \mathrm{~g}$ of 12 .

Hydroxyester 11: $\mathrm{R} f=0.06\left(20 \% \mathrm{MeOH} / \mathrm{CH}_{2} \mathrm{Cl}_{2}\right)$; IR (thin film) 3420, 2937, 2850, 1941, 1733 $\mathrm{cm}^{-1}$; ${ }^{1} \mathrm{H}$ NMR $\left(500 \mathrm{MHz}, \mathrm{CDCl}_{3}\right) \delta 4.31$ (ddd, $\left.J=4.7,4.7,2.5 \mathrm{~Hz}, 1 \mathrm{H}\right), 3.71(\mathrm{~s}, 3 \mathrm{H}), 3.68(\mathrm{dd}, J=$ 8.1, $4.6 \mathrm{~Hz}, 1 \mathrm{H}), 3.20(\mathrm{~m}, 2 \mathrm{H}), 3.08(\mathrm{ddd}, J=9.6,7.1,2.7 \mathrm{~Hz}, 1 \mathrm{H}), 2.67(\mathrm{~m}, 1 \mathrm{H}), 2.59$ (ddd, $J=$ 9.5, 9.5, 6.3 Hz, $1 \mathrm{H}), 2.24(\mathrm{~m}, 3 \mathrm{H}), 2.10(\mathrm{~m}, 1 \mathrm{H}), 1.97(\mathrm{~m}, 1 \mathrm{H}) ;{ }^{13} \mathrm{C} N M R\left(125 \mathrm{MHz}, \mathrm{CDCl}_{3}\right)$ $\delta$ 175.0, 72.3, 70.9, 55.4, 52.4, 52.2, 42.0, 37.0, 31.8; HRMS calcd for $\mathrm{C}_{9} \mathrm{H}_{15} \mathrm{NO}_{3}$ 186.1130, found 186.1130 .

Lactone 12: $\mathrm{R} f=0.33\left(20 \% \mathrm{MeOH} / \mathrm{CH}_{2} \mathrm{Cl}_{2}\right)$; IR (thin film) 3408, 2929, $1772 \mathrm{~cm}^{-1} ;{ }^{1} \mathrm{H}$ NMR (500 $\mathrm{MHz}_{\mathrm{CDCl}}$ ) $\delta 5.00$ (ddd, $J=6.6,5.2,1.4 \mathrm{~Hz}, 1 \mathrm{H}$ ), 4.14 (dd, $J=7.6,5.4 \mathrm{~Hz}, 1 \mathrm{H}$ ), 3.15 (dddd, $J$ $=12.8,9.2,5.2,4.5 \mathrm{~Hz}, 1 \mathrm{H}), 3.12-3.05(\mathrm{~m}, 2 \mathrm{H}), 2.81$ (ddd, $J=11.1,6.6,6.6 \mathrm{~Hz}, 1 \mathrm{H}), 2.67$ (ddd, $J=10.0,10.0,6.2 \mathrm{~Hz}, 1 \mathrm{H}), 2.40-2.29(\mathrm{~m}, 2 \mathrm{H}), 2.29-2.22(\mathrm{~m}, 1 \mathrm{H}), 2.18-2.10(\mathrm{~m}, 1 \mathrm{H}) ;{ }^{13} \mathrm{C} \mathrm{NMR}$ $\left(125 \mathrm{MHz}, \mathrm{CDCl}_{3}\right) \delta 179.7,83.1,70.9,53.8,51.3,45.4,33.7,31.3$; HRMS calcd for $\mathrm{C}_{8} \mathrm{H}_{11} \mathrm{NO}_{2}$ 154.0868, found: 154.0862 .

(士)-Turneforcidine. To a solution of hydroxyester $11(0.015 \mathrm{~g}, 0.08 \mathrm{mmol})$ in THF $(7.9 \mathrm{~mL})$ at 0 ${ }^{\circ} \mathrm{C}$ was added $\mathrm{LiAlH}_{4}(0.40 \mathrm{~mL}, 0.39 \mathrm{mmol}, 1.0 \mathrm{M}$ in THF). The resulting solution was then heated to $65{ }^{\circ} \mathrm{C}$ for $4 \mathrm{~h}$ at which time it was cooled to $0{ }^{\circ} \mathrm{C}$ and quenched by slow addition of a $2: 1$ mixture of sodium sulfate decahydrate/celite. The solution was allowed to warm to it and stirred for an additional $4 \mathrm{~h}$, followed by filtration through celite and washing with a $1: 1 \mathrm{MeOH} / \mathrm{CH}_{2} \mathrm{Cl}_{2}$ solution. Concentration of the filtrate under reduced pressure yielded $0.012 \mathrm{~g}(95 \%)$ of turneforcidine as an oil, judged to by pure by TLC and NMR analysis. IR (thin film) 3422, 3020, 2927, 2066, $1652 \mathrm{~cm}^{-1}$; ${ }^{1} \mathrm{H}$ NMR (500 MHz, $\mathrm{CDCl}_{3}$ ) $\delta 4.61$ (br s, $\left.2 \mathrm{H}\right), 4.32$ (dd, $J=10.0,5.4 \mathrm{~Hz}$, $1 \mathrm{H}), 3.80$ (dd, $J=10.0,4.5 \mathrm{~Hz}, 1 \mathrm{H}), 3.41(\mathrm{dd}, J=9.8,9.8 \mathrm{~Hz}, 1 \mathrm{H}), 3.32(8.3,5.6 \mathrm{~Hz}, 1 \mathrm{H}), 3.20$ (ddd, $J=9.6,7.3,2.2 \mathrm{~Hz}, 1 \mathrm{H}$ ), 3.03 (ddd, $J=10.4,6.9,5.0 \mathrm{~Hz}, 1 \mathrm{H}$ ), 2.68 (ddd, $J=10.2,8.5,6.5$ $\mathrm{Hz}, 1 \mathrm{H}), 2.61$ (ddd, $J=10.3,10.3,6.0 \mathrm{~Hz}, 1 \mathrm{H}), 2.49(\mathrm{~m}, 1 \mathrm{H}), 2.03(\mathrm{~m}, 2 \mathrm{H}), 1.92(\mathrm{~m}, 1 \mathrm{H}), 1.60$ (dddd, $J=12.1,10.6,10.6,7.3 \mathrm{~Hz}, 1 \mathrm{H}) ;{ }^{13} \mathrm{C}$ NMR (125 MHz, $\mathrm{CDCl}_{3}$ ), $\delta 74.3,71.5,65.3,55.2$, 52.3, 40.5, 35.4, 31.0.

( \pm )-Platynecine. To a solution of lactone $12(0.006 \mathrm{~g}, 0.03 \mathrm{mmol})$ in THF $(2.0 \mathrm{~mL})$ at $0{ }^{\circ} \mathrm{C}$ was added $\mathrm{LiAlH}_{4}(0.12 \mathrm{~mL}, 0.12 \mathrm{mmol}, 1.0 \mathrm{M}$ in THF). The resulting solution was then heated to 65 ${ }^{\circ} \mathrm{C}$ for $4 \mathrm{~h}$ at which time it was cooled to $0{ }^{\circ} \mathrm{C}$ and quenched by slow addition of a $5 \%$ aqueous 
solution of THF. The solution was then passed through a celite plug with $5 \% \mathrm{MeOH} / \mathrm{CHCl}_{3}$. Purification of the filtrate was accomplished by column chromatography in a pipet eluting with a 5:5:1 $\mathrm{CHCl}_{3} / \mathrm{MeOH} / \mathrm{NH}_{4} \mathrm{OH}$. Product-containing fractions were concentrated under reduced pressure to provide $0.006 \mathrm{~g}(97 \%)$ of platynecine as an oil: IR (thin film) 3429, 3019, 2926, 2854, $2089 \mathrm{~cm}^{-1}$; ${ }^{1} \mathrm{H}$ NMR (500 MHz, CD $\left.{ }_{3} \mathrm{OD}\right) \delta 4.29(\mathrm{~m}, 1 \mathrm{H}), 3.95$ (dddd, $J=11.0,11.0,11.0,5.9 \mathrm{~Hz}$, 2H), 3.38 (dd, $J=8.0,3.3 \mathrm{~Hz}, 1 \mathrm{H}), 3.31(\mathrm{~m}, 1 \mathrm{H}), 3.17$ (ddd, $J=10.7,10.7,7.1 \mathrm{~Hz}, 1 \mathrm{H}), 2.93$ (ddd, $J=11.1,11.1,7.1 \mathrm{~Hz}, 1 \mathrm{H}$ ), 2.87 (ddd, $J=11.3,9.1,2.5 \mathrm{~Hz}, 1 \mathrm{H}$ ), $2.47(\mathrm{~m}, 1 \mathrm{H}), 2.03(\mathrm{~m}$, $1 \mathrm{H}$ ), $1.91(\mathrm{~m}, 2 \mathrm{H}), 1.77$ (dddd, $J=9.6,7.1,7.1,2.5 \mathrm{~Hz}, 1 \mathrm{H}) ;{ }^{13} \mathrm{C}$ NMR $\left(125 \mathrm{MHz}, \mathrm{CD}_{3} \mathrm{OD}\right) \delta$ 73.1, 72.9, 61.7, 56.7, 55.1, 45.1, 37.4, 29.0; HRMS calcd for $\mathrm{C}_{8} \mathrm{H}_{15} \mathrm{NO}_{2}$ 158.1180, found 158.1176.

Benzyl 1-(3'-Oxo-4'-diazo)butylpyrrolidine-2-carboxylate 1b. To proline benzyl ester hydrochloride $(0.778 \mathrm{~g}, 3.22 \mathrm{mmol})$ was added freshly prepared bromide $30(0.285 \mathrm{~g}, 1.61 \mathrm{mmol})$. The flask was then flushed with argon and acetonitrile $(6.4 \mathrm{~mL})$ was added followed by triethylamine $(0.90 \mathrm{~mL}, 6.4 \mathrm{mmol})$ via syringe and the resulting mixture was allowed to stir at 35 ${ }^{\circ} \mathrm{C}$ for $12 \mathrm{~h}$. The mixture was concentrated under reduced pressure and then purified by flash column chromatography on a $2.0 \mathrm{~cm} \times 11.0 \mathrm{~cm}$ column eluting with $50 \%$ EtOAc/hexanes and collecting $4 \mathrm{~mL}$ fractions. The product-containing fractions were collected and concentrated under reduced pressure to provide $0.461 \mathrm{~g}(95 \%)$ of diazo compound 35 as a yellow oil: $R_{f}=0.63$ $\left(10 \% \mathrm{MeOH} / \mathrm{CH}_{2} \mathrm{Cl}_{2}\right)$; IR (neat) 3090, 3034, 2956, 2818, 2102, 1960, 1815, 1743, $1648 \mathrm{~cm}^{-1} ;{ }^{1} \mathrm{H}$

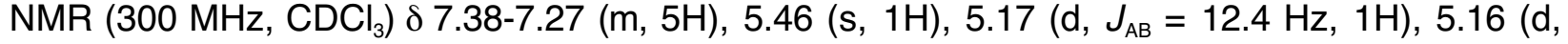
$J_{\mathrm{AB}}=12.4 \mathrm{~Hz}, 1 \mathrm{H}$ ), 3.23 (dd, $J=8.6,5.5 \mathrm{~Hz}, 1 \mathrm{H}$ ), 3.15 (ddd, $J=7.8,7.8,3.3 \mathrm{~Hz}, 1 \mathrm{H}$ ), 3.01 (ddd, $J=12.4,7.6,7.6 \mathrm{~Hz}, 1 \mathrm{H}$ ), 2.73 (ddd, $J=13.3,6.8,6.8 \mathrm{~Hz}, 1 \mathrm{H}$ ), 2.53-2.43 (m, 2H), 2.37 (app q, $J=8.1 \mathrm{~Hz}, 1 \mathrm{H}), 2.20-2.06(\mathrm{~m}, 1 \mathrm{H}), 2.00-1.75(\mathrm{~m}, 3 \mathrm{H}) ;{ }^{13} \mathrm{C}$ NMR $\left(75 \mathrm{MHz}, \mathrm{CDCl}_{3}\right) \delta$ 193.9, 174.1, 136.0, 128.8, 128.5, 128.5, 66.6, 66.1, 54.9, 53.6, 50.2, 40.2, 29.6, 23.4; Anal. Calcd for $\mathrm{C}_{16} \mathrm{H}_{19} \mathrm{~N}_{3} \mathrm{O}_{3}$ : C, 63.77; $\mathrm{H}, 6.36 ; \mathrm{N}, 13.94$. Found: C 63.81; $\mathrm{H}, 6.47 ; \mathrm{N}, 13.82$.

Indolizidines $\mathbf{3 b}$ and $\mathbf{4 b}$. To a stirring solution of $\mathrm{Rh}_{2}(\mathrm{OAc})_{4}(0.005 \mathrm{~g}, 0.010 \mathrm{mmol})$ in benzene $(4.0 \mathrm{~mL})$ at $85^{\circ} \mathrm{C}$ was added a solution of diazoketone $35(0.063 \mathrm{~g}, 0.208 \mathrm{mmol})$ in benzene $(0.40$ $\mathrm{mL})$ dropwise over $5 \mathrm{~min}$. Upon complete addition, the syringe was washed with benzene $(0.2$ $\mathrm{mL}$ ) and added directly to the reaction. The resulting solution was allowed to cool to rt and concentrated under reduced pressure to provide a brown oil. The oil was immediately purified b y flash column chromatography on a $1.5 \mathrm{~cm} \times 15 \mathrm{~cm}$ column eluting with $30 \mathrm{~mL}$ each of $20 \%, 40 \%$, $60 \%$, and $100 \%$ EtOAc/Hexanes followed by $50 \mathrm{~mL}$ of $4 \% \mathrm{MeOH} / \mathrm{CH}_{2} \mathrm{Cl}_{2}$ to yield $0.048 \mathrm{~g}(85 \%)$ of $\mathbf{3 b}$ and $\mathbf{4 b}$ as a brown oil (mixture of diastereomers: $\mathrm{R}_{f}=0.69\left(10 \% \mathrm{MeOH} / \mathrm{CH}_{2} \mathrm{Cl}_{2}\right) ;{ }^{1} \mathrm{H} \mathrm{NMR}$ $\left(300 \mathrm{MHz}, \mathrm{CDCl}_{3}\right) \delta$ 7.40-7.27 (m, 10H), 5.19-5.10 (m, 4H), $3.62(\mathrm{dd}, J=6.2,6.2 \mathrm{~Hz}, 1 \mathrm{H}), 3.52$ (ddd, $J=8.6,4.9,4.9 \mathrm{~Hz}, 1 \mathrm{H}), 3.42(\mathrm{dd}, J=3.6,3.6 \mathrm{~Hz}, 1 \mathrm{H}) 3.25-3.19(\mathrm{~m}, 1 \mathrm{H}), 3.14-3.05(\mathrm{~m}$, $3 \mathrm{H})$, 3.04-2.92 (m, $1 \mathrm{H}), 2.73-2.54(\mathrm{~m}, 4 \mathrm{H}), 2.35(\mathrm{ddd}, J=16.3,16.3,8.1 \mathrm{~Hz}, 1 \mathrm{H}), 2.24-2.04(\mathrm{~m}$, $3 \mathrm{H})$, 2.03-1.74 (m, 8H); ${ }^{13} \mathrm{C}$ NMR $\left(75 \mathrm{MHz} \mathrm{CDCl}_{3}\right) \delta 208.2,208.0,174.1,174.0,136.1,136.1$, $128.7,128.5,128.5,128.4,128.4,128.4,66.5,66.4,66.0,66.0,64.7,64.7,62.7,62.6,53.6,49.1$, $49.1,39.7,29.6,29.5,23.8,23.4$.

${ }^{1}$.Aldrich Chemical Co., Milwaukee, WI: Technical Information Bulletin No. AL-113.

${ }^{2}$ Phillips, B. A.; Cromwell, N. H. J. Heterocycl. Chem. 1973, 10, 795-799.

${ }^{3}$ (a) Clark, J. S.; Hodgson, P. B.; Goldsmith, M. D.; Street, L. J. J. Chem. Soc., Perkin Trans. 12001 , 3312-3324. (b) Rosenquist, N. R.; Chapman, O. L. J. Org. Chem. 1976, 41, 3326-3327.

${ }^{4}$ (a) Hudlicky, T.; Seoane, G.; Lovelace, T. C. J. Org. Chem. 1988, 53, 2094-2099. (b) Chamberlin, A. R.; Chung, J. Y. L. J. Org. Chem. 1985, 50, 4425-4431. 


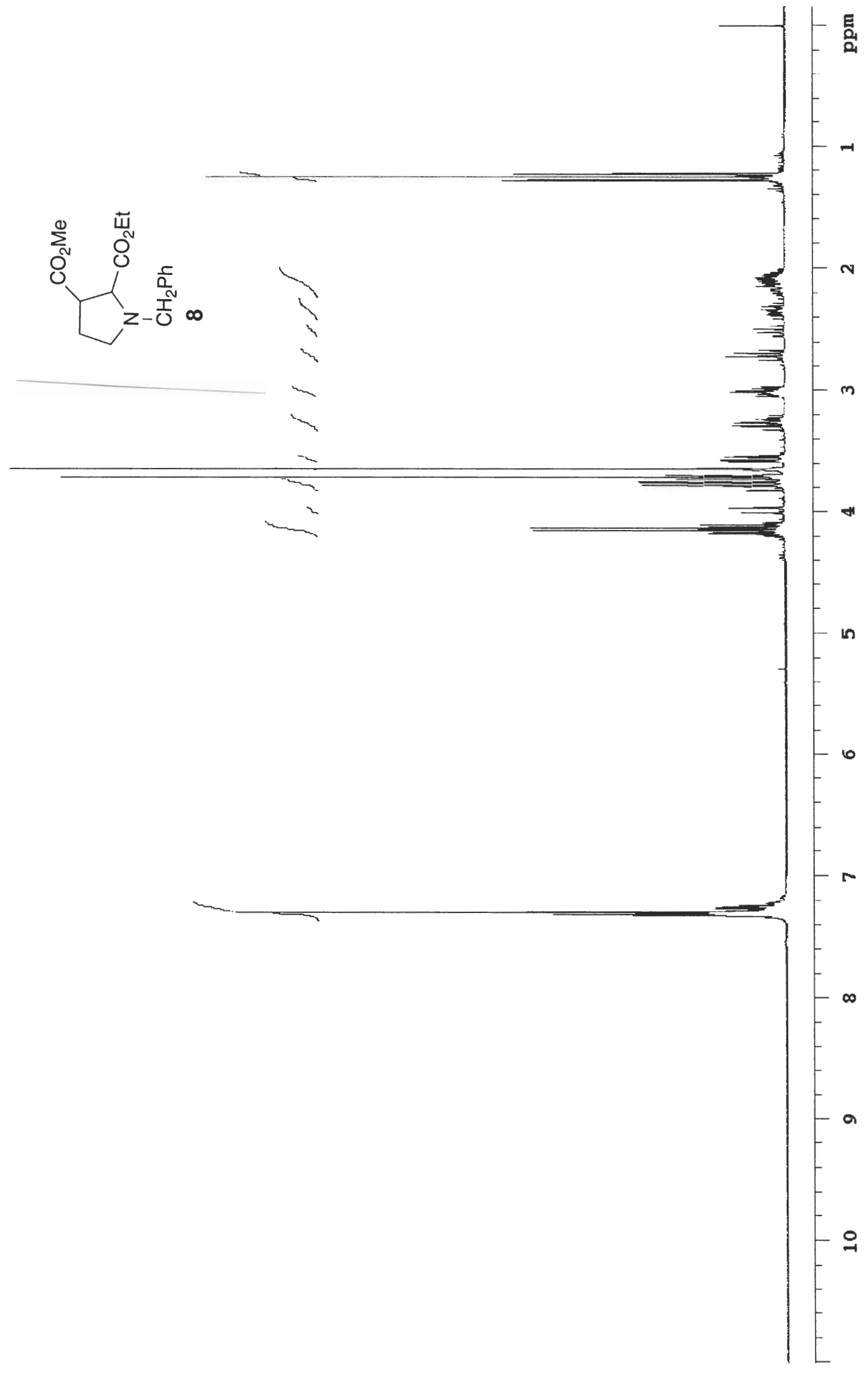

S- 5 

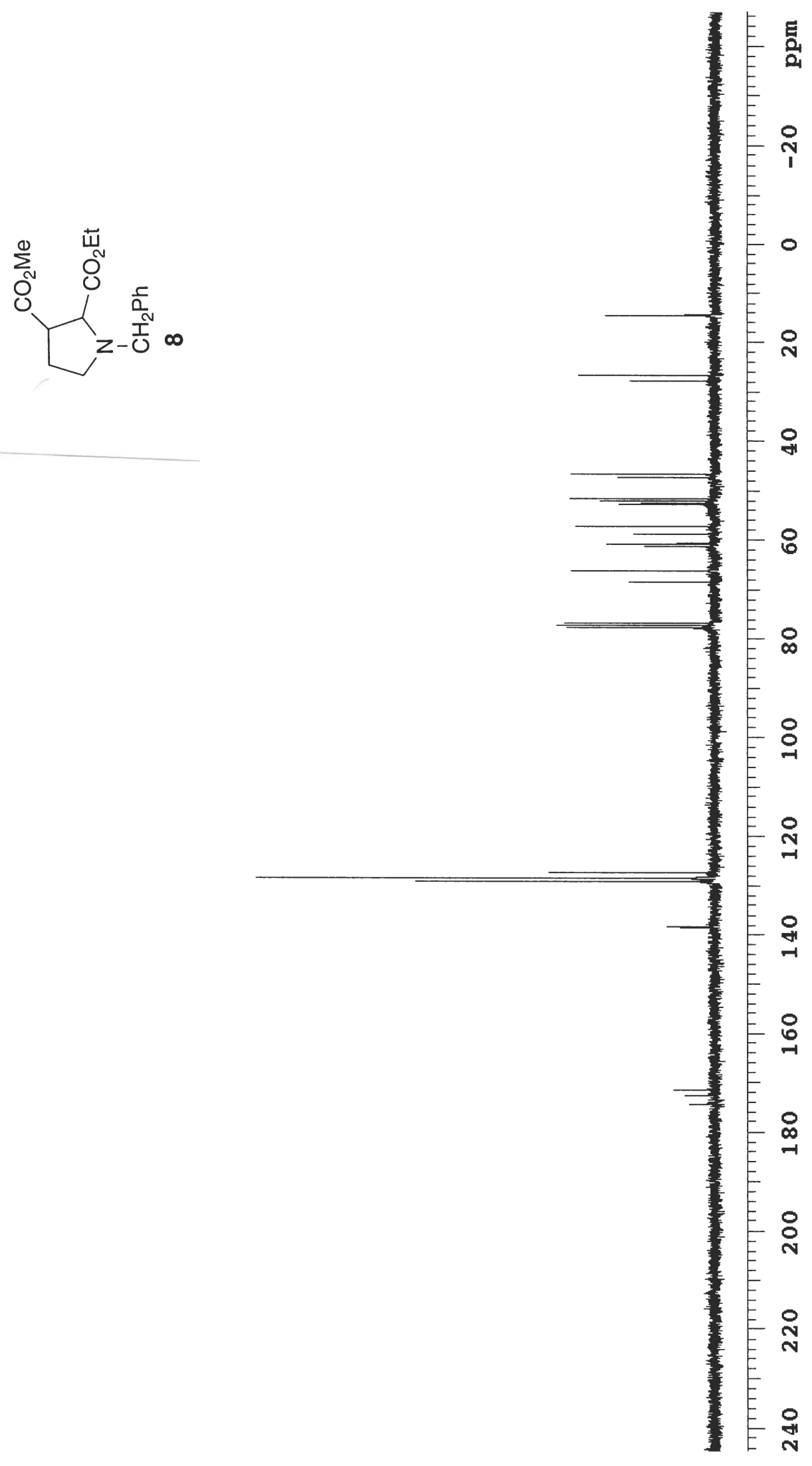

S- 6 

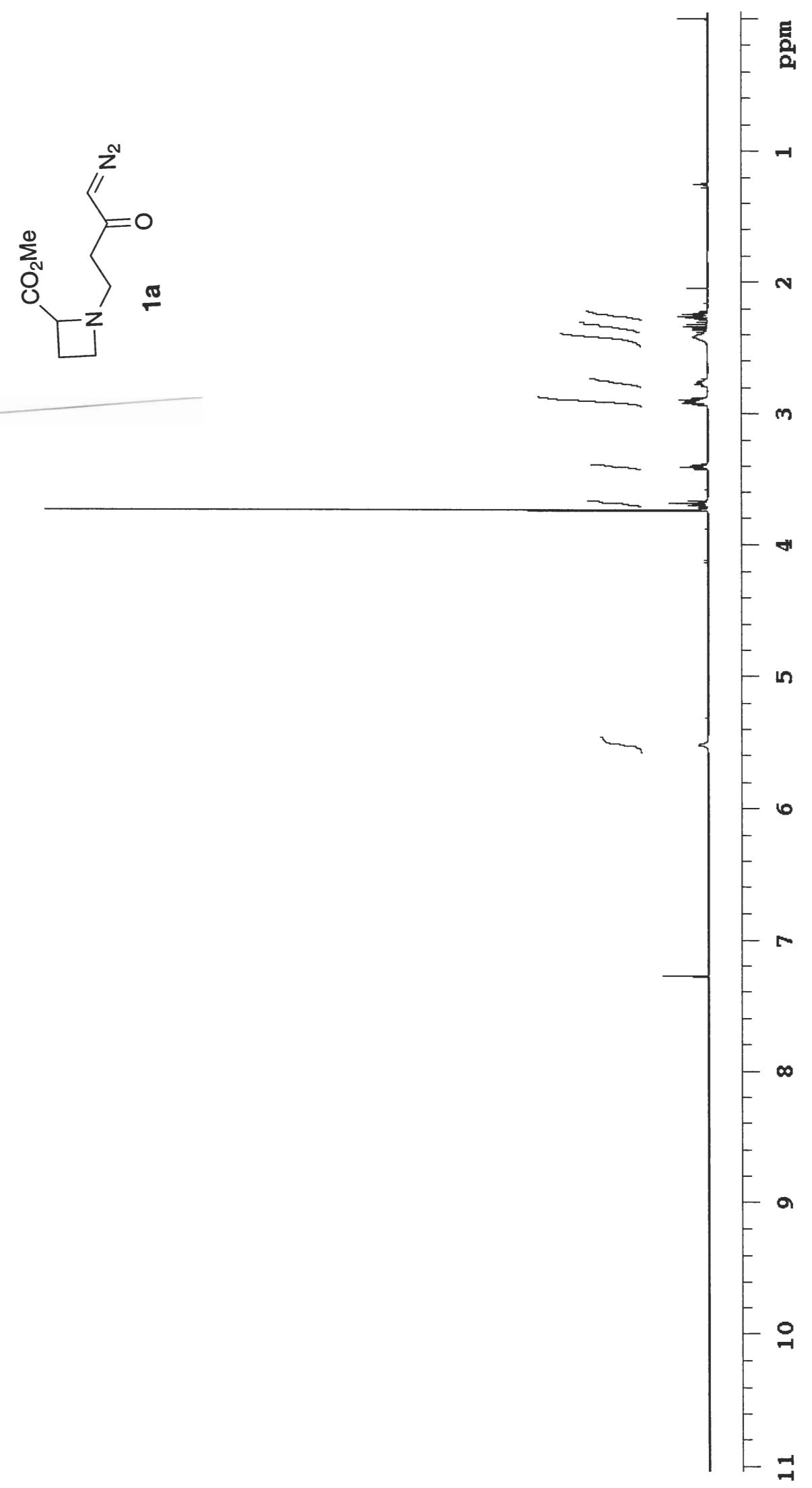

S- 7 


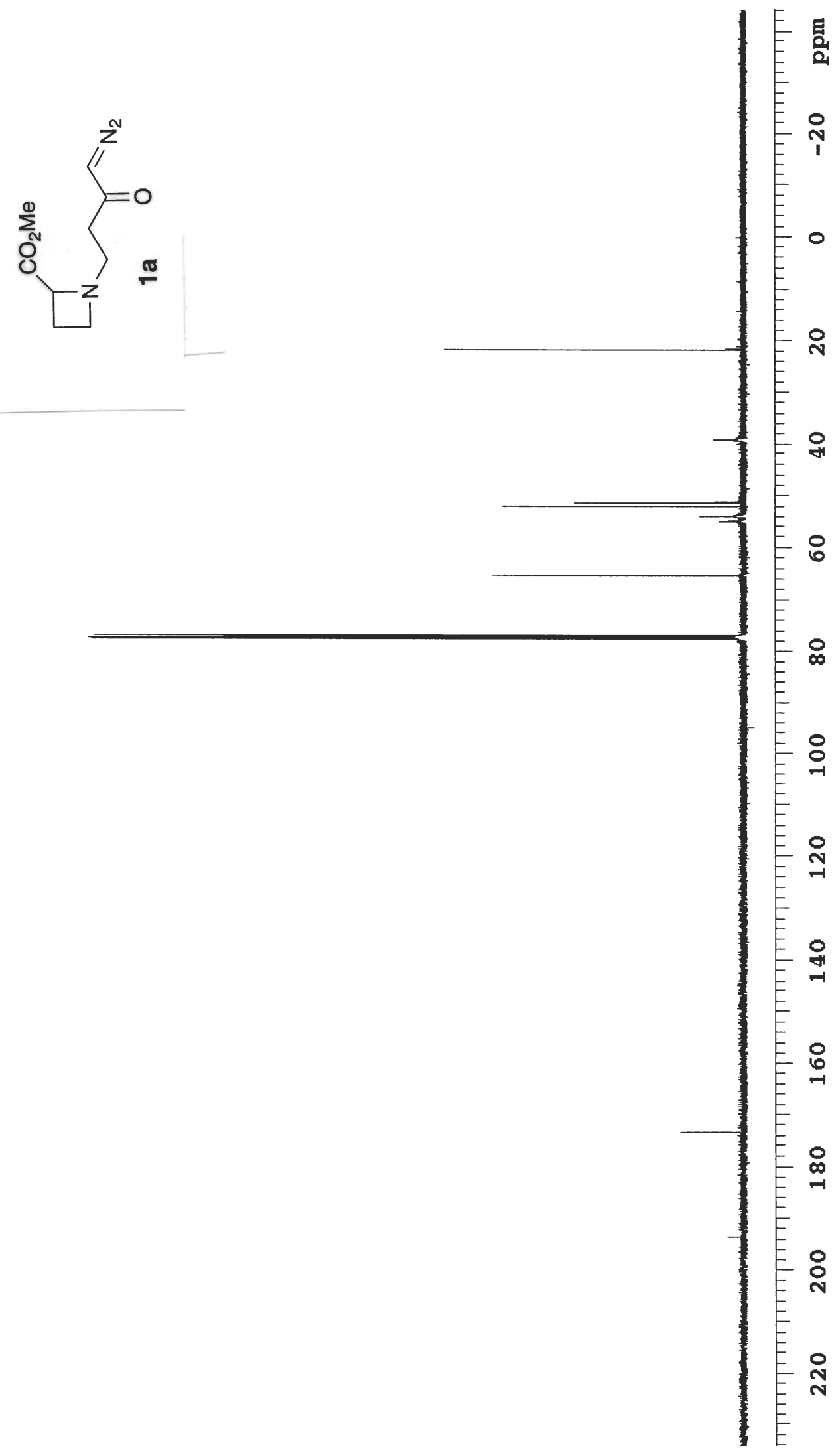

S- 8 


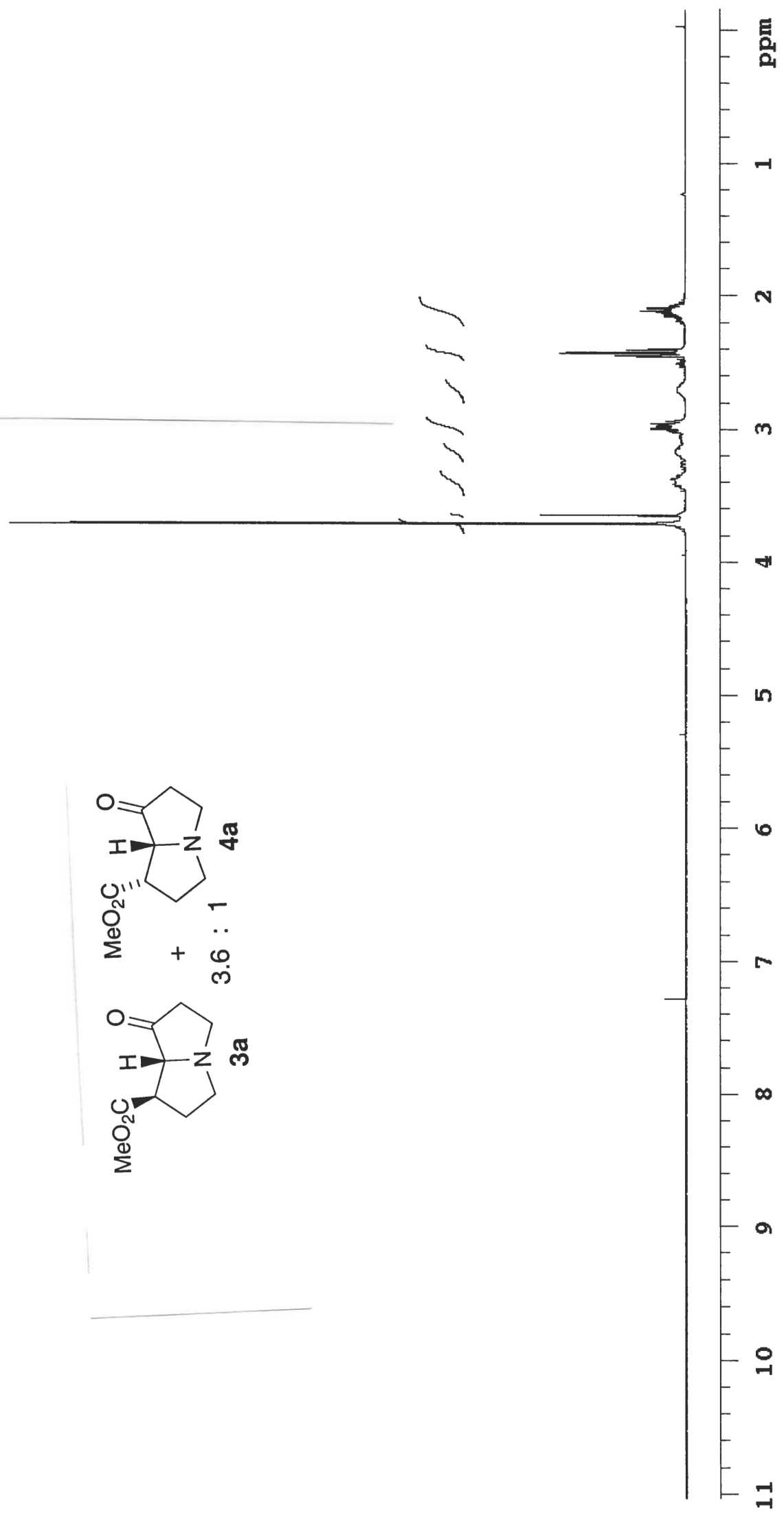

S- 9 


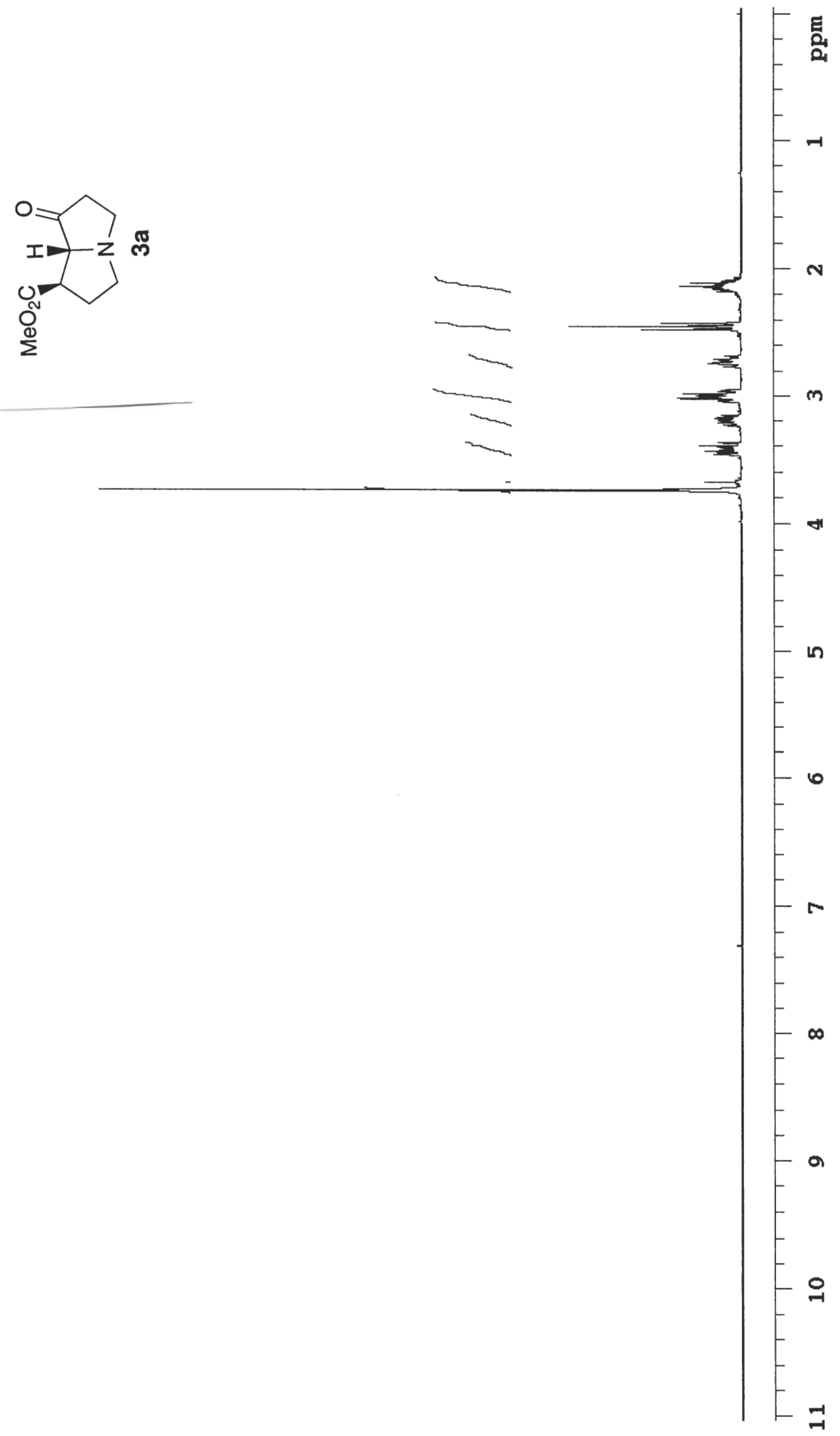



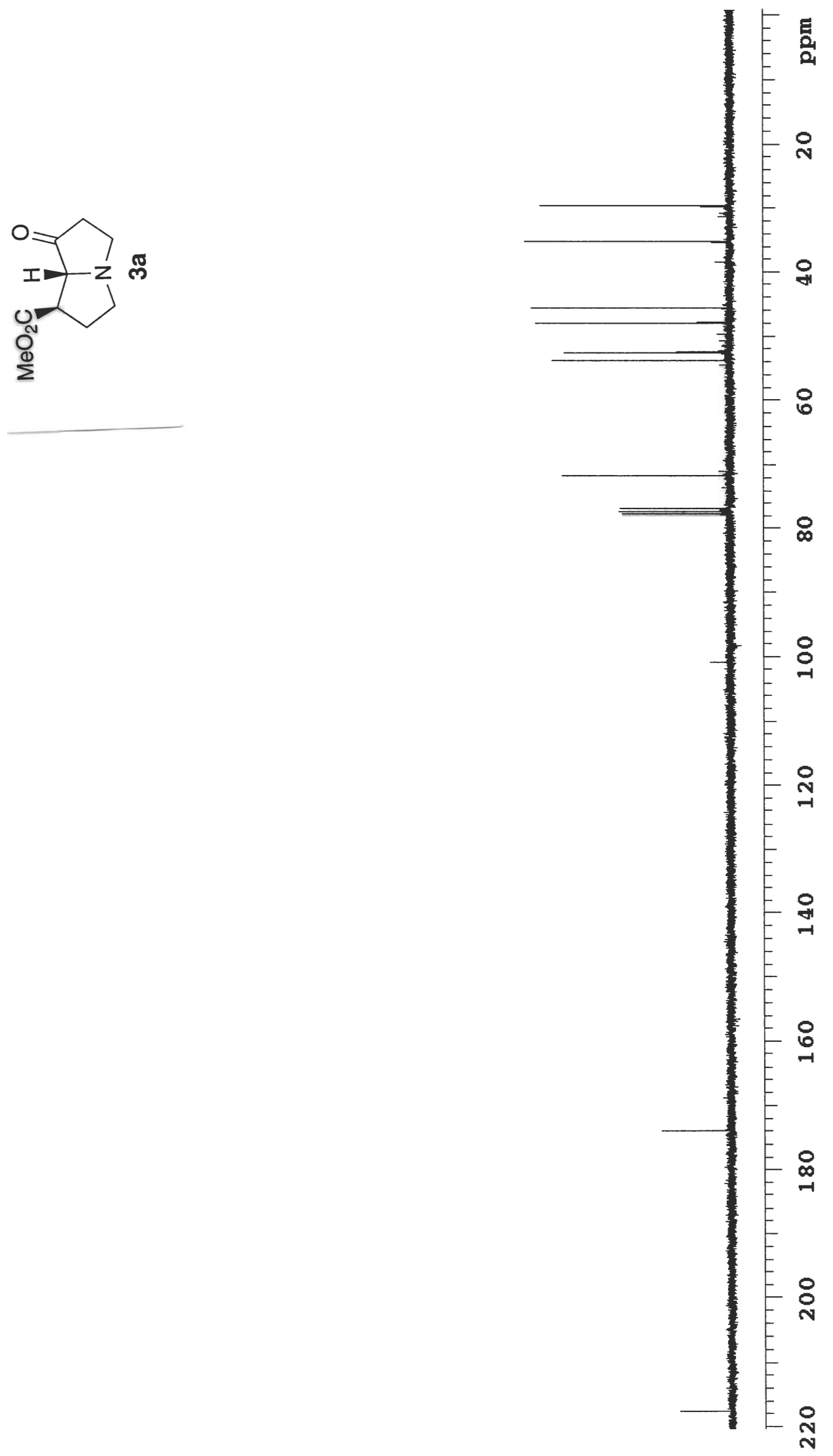

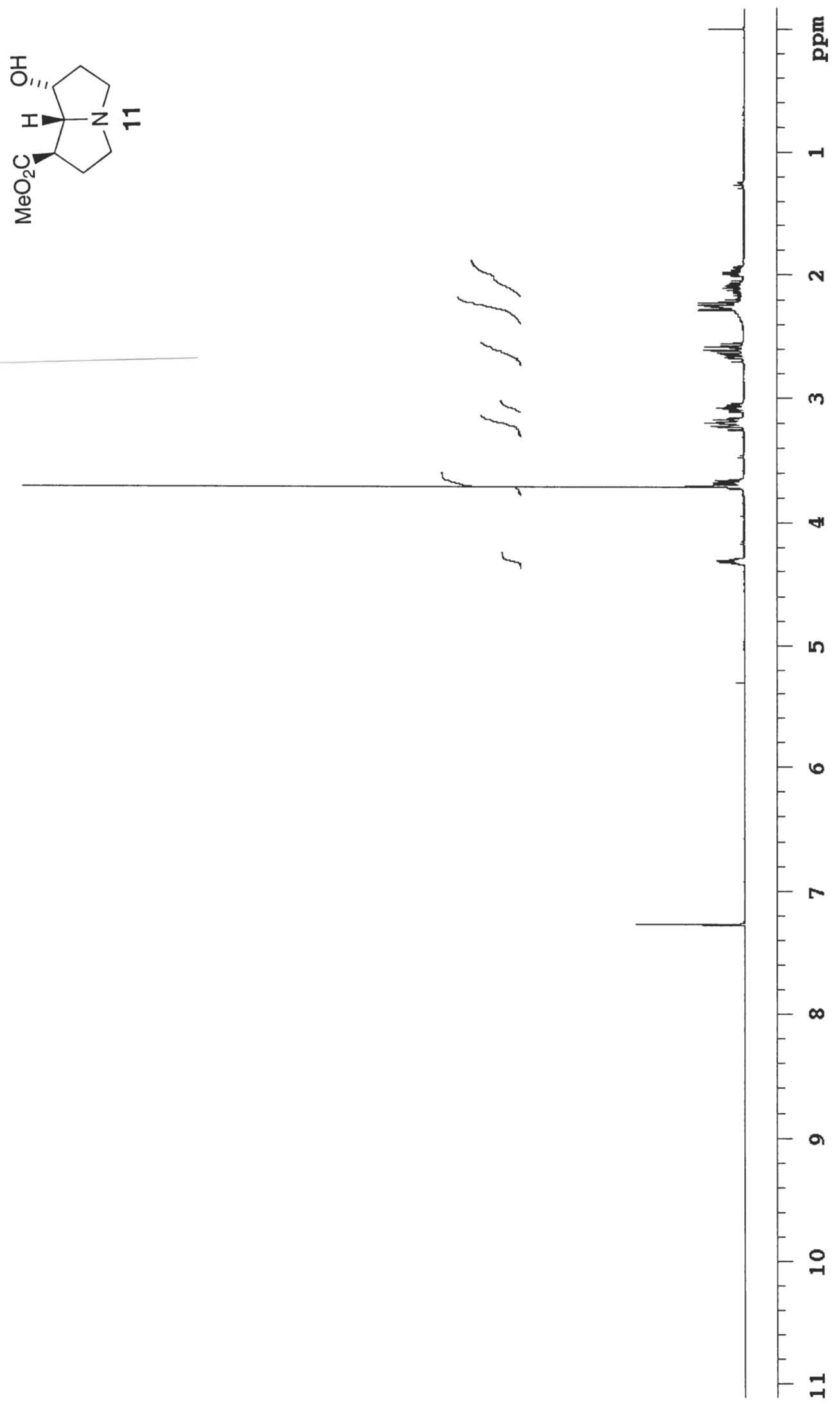


$$
4
$$



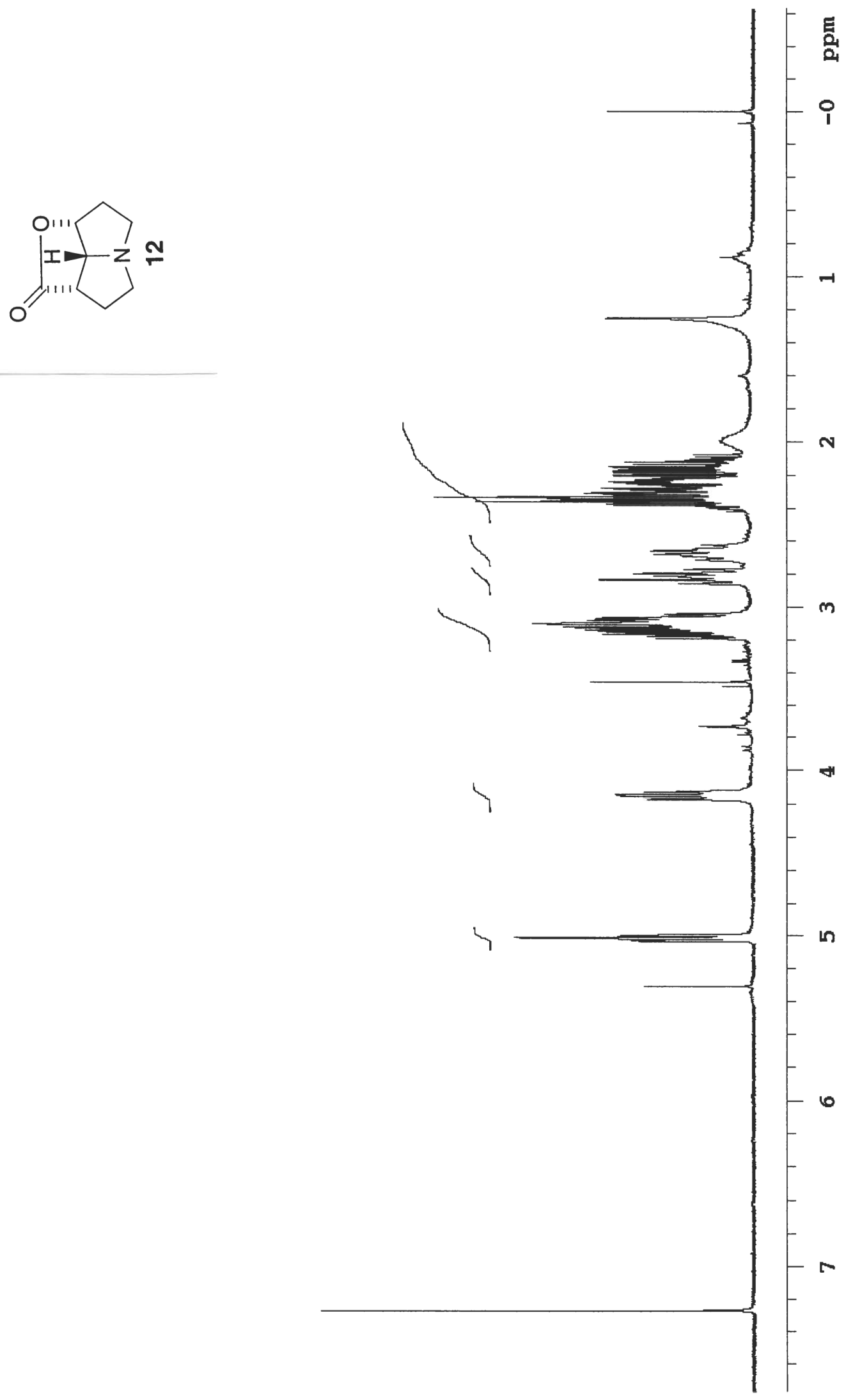


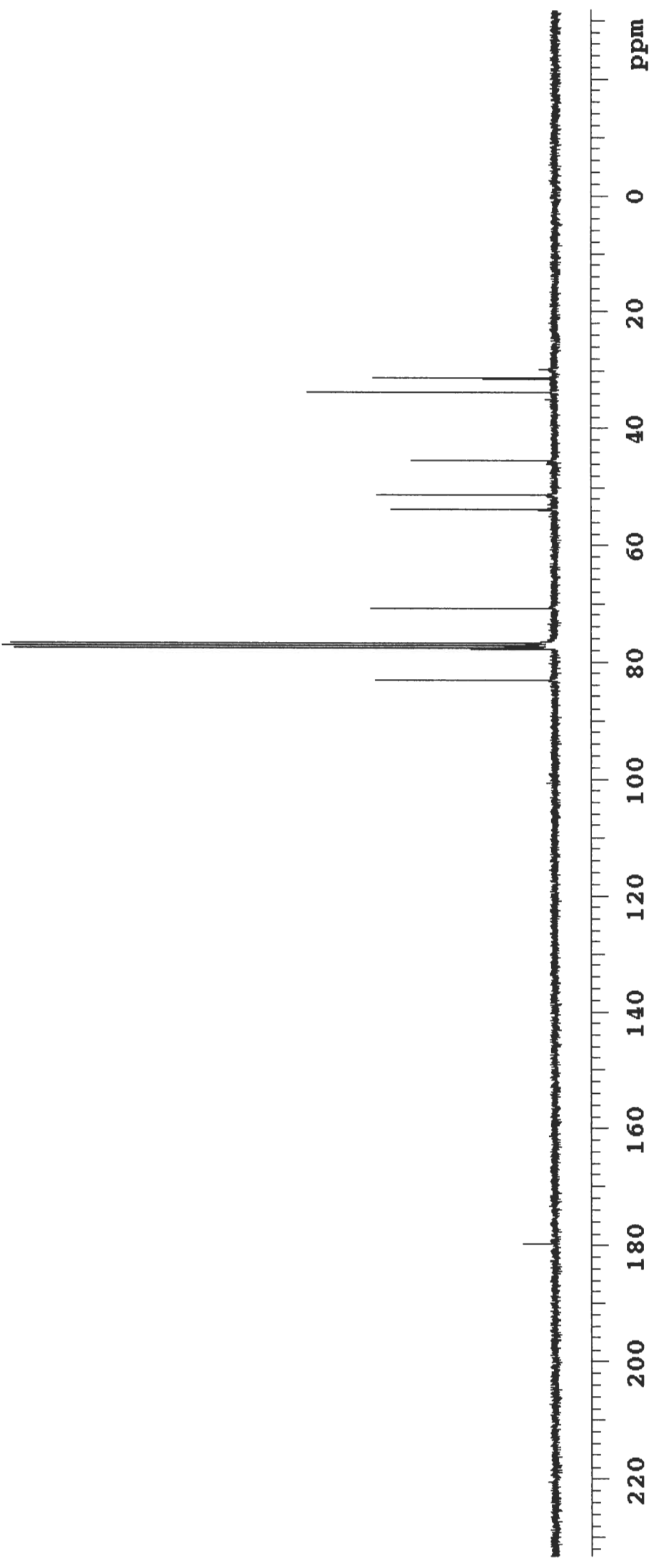



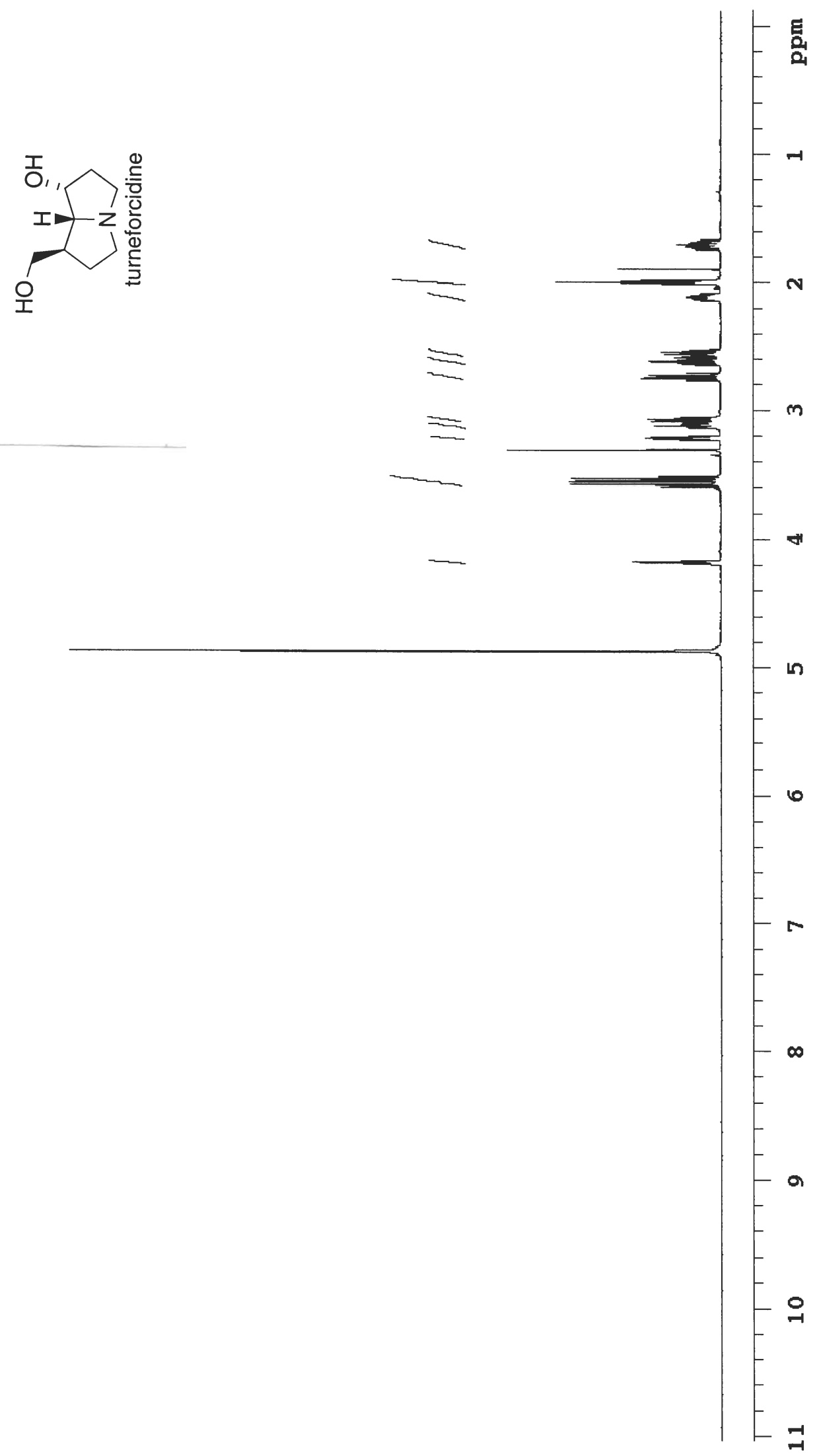

S-16 


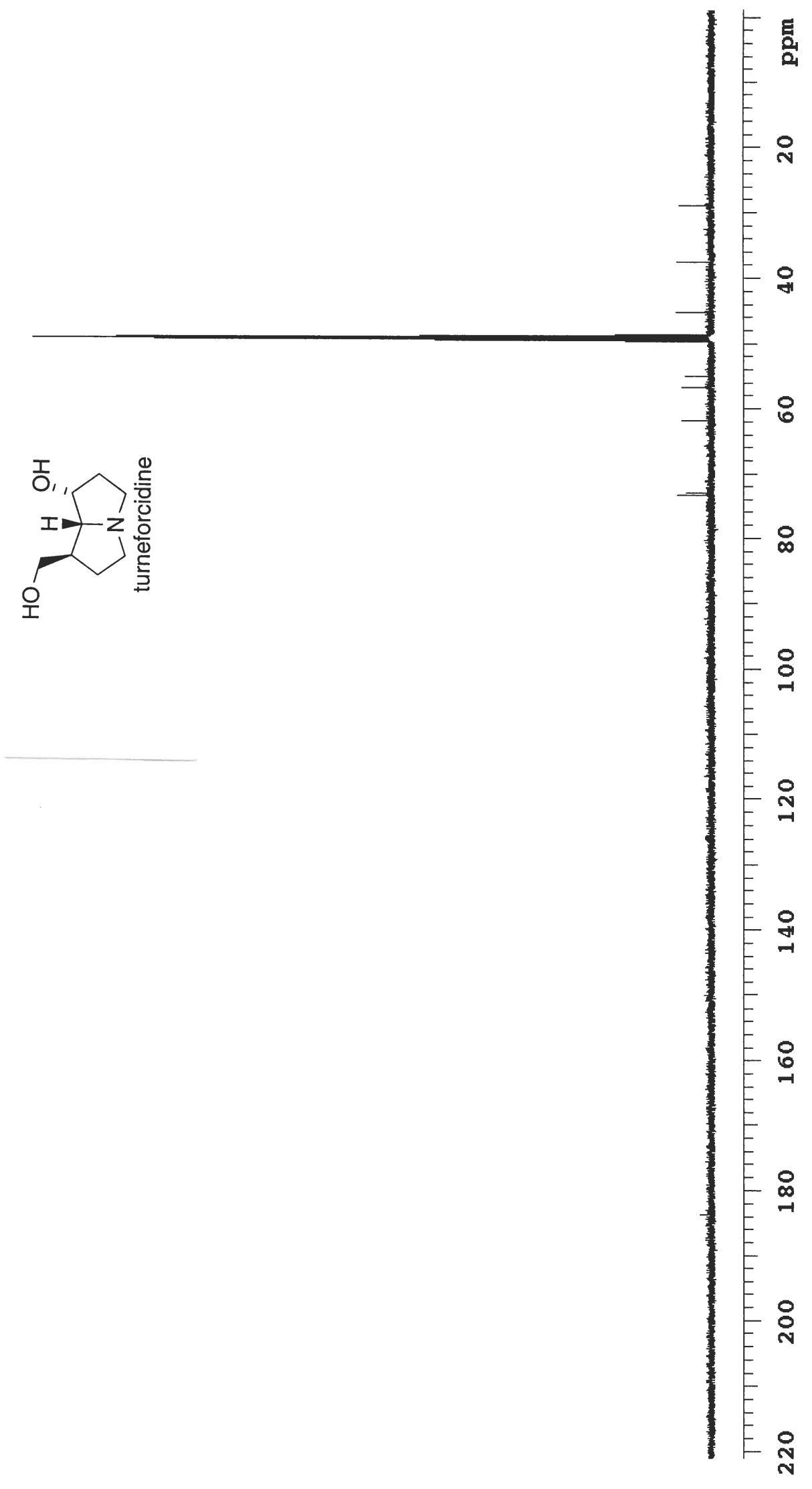



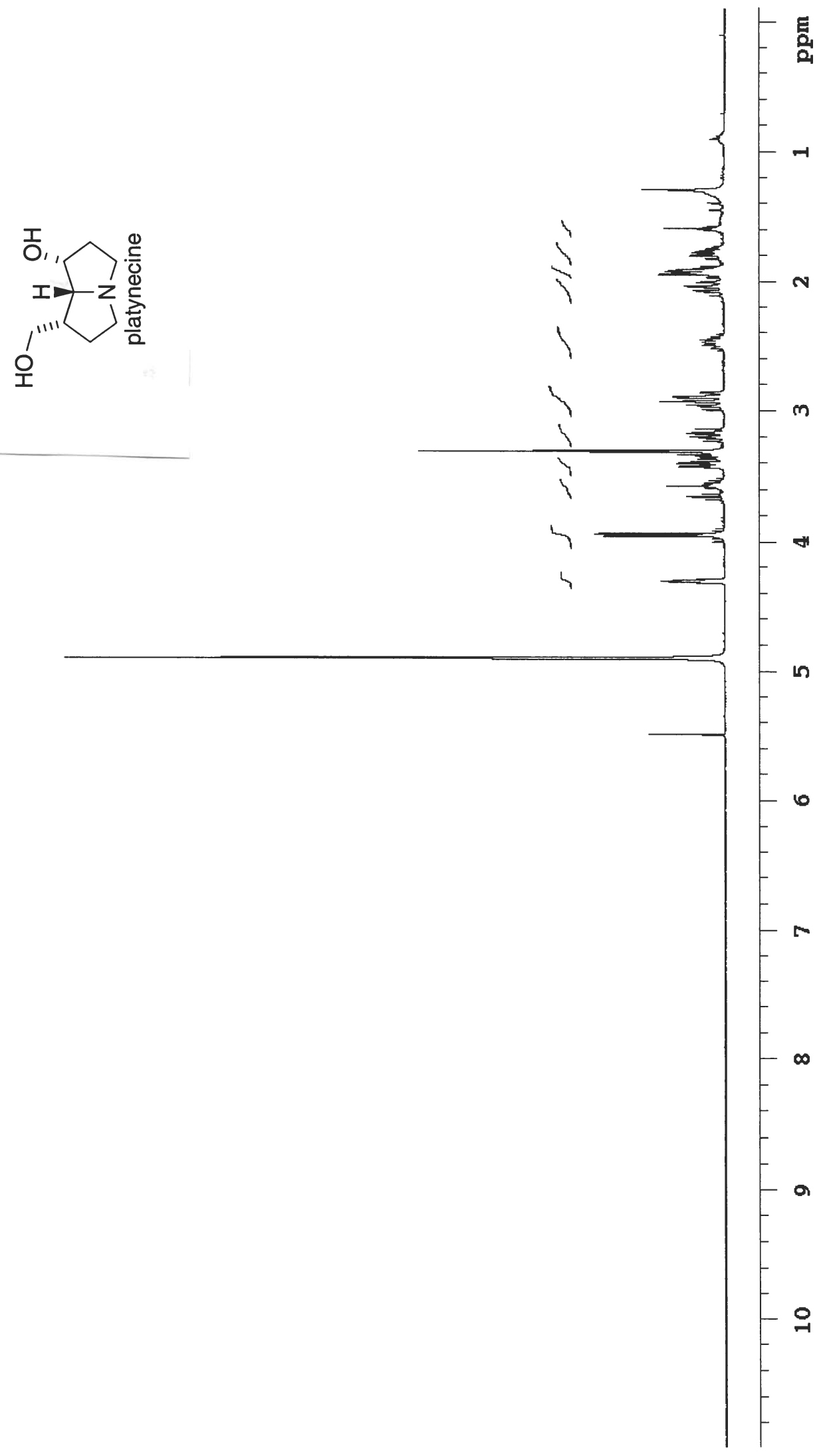


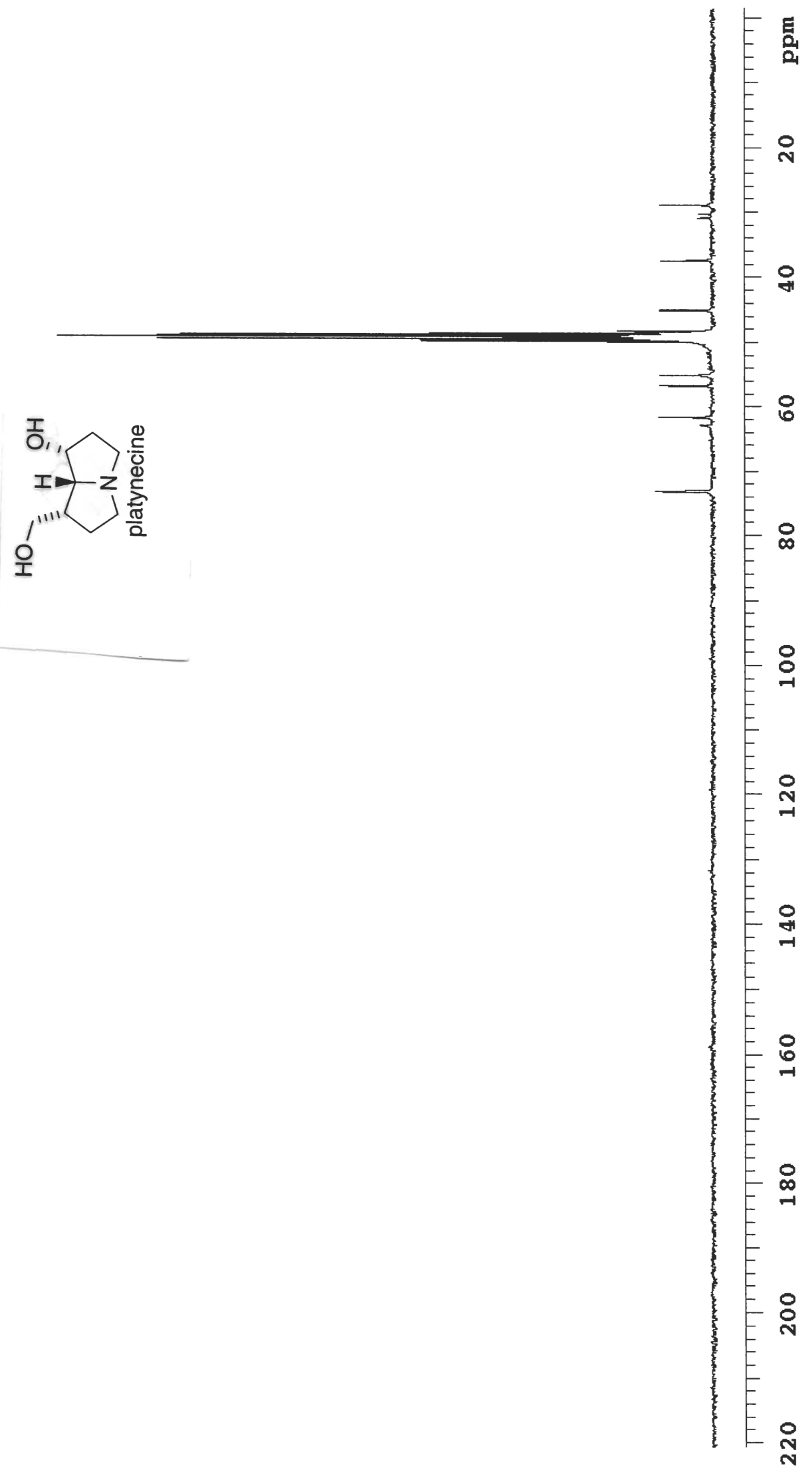



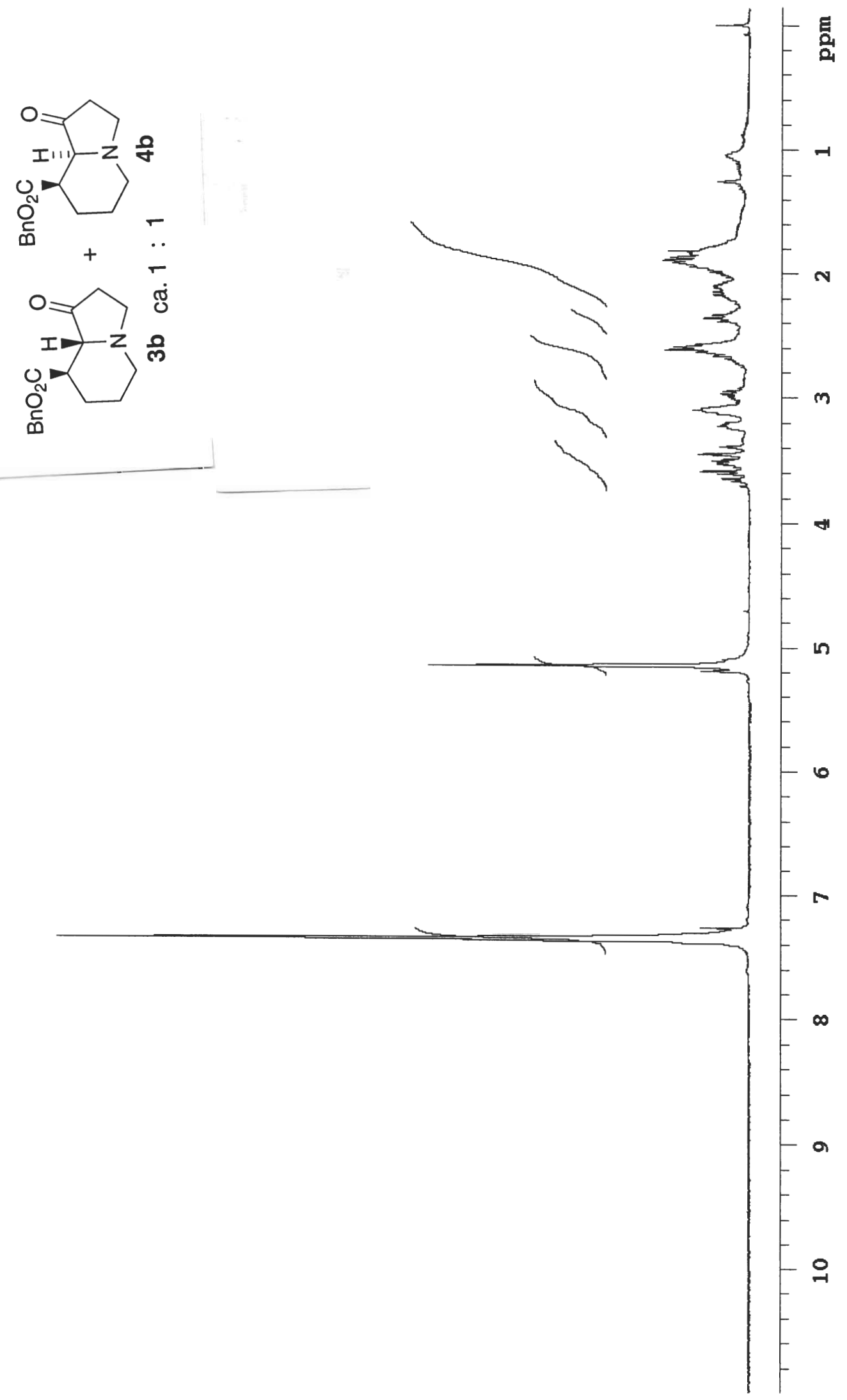


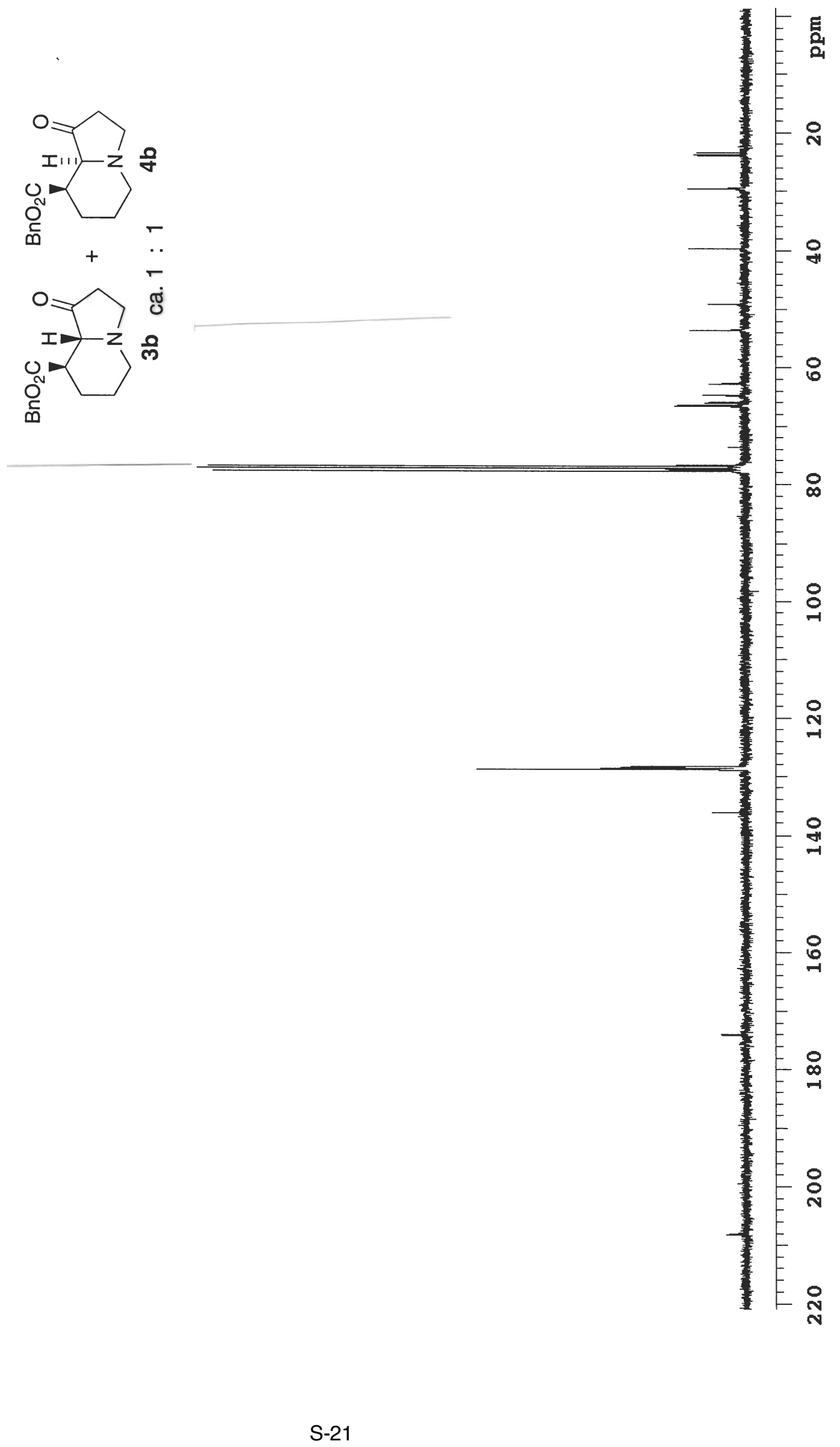

